\title{
Cytokines, chemokines and leukotrienes profile and signature analysis in HTLV-1 infection as an evidence of disease progression
}

\author{
Ana LB Starling ${ }^{1}$, Denise U Gonçalves ${ }^{1 *}$, Vanessa Peruhype-Magalhães ${ }^{2}$, Jordana Coelho-dos-Reis ${ }^{2}$, \\ José R Lambertucci', Ludimila Labanca ${ }^{1}$, Silvio R Souza Pereira ${ }^{1}$, Marina L Martins ${ }^{3}$, João G Ribas ${ }^{4}$, \\ Andréa Teixeira-Carvalho ${ }^{2}$, Bruno C Trindade ${ }^{2}$, Lucia H Faccioli', Anna BF Carneiro-Proietti ${ }^{3}$, \\ Olindo A Martins-Filho ${ }^{5}$
}

From 16th International Conference on Human Retroviruses: HTLV and Related Viruses Montreal, Canada. 26-30 June 2013

\section{Background}

A cross-sectional study evaluated the cytokines, chemokines and leukotrienes profiles as possible biomarkers of progression to HTLV-1 associated myelopathy (HAM).

\section{Methods}

Serum samples from 21 healthy blood donors (HBD), 27 asymptomatic carriers (ASC), 32 possible HAM (pHAM) and 28 HAM individuals were tested for cytokines (IL-6, IFN- $\gamma$, TNF- $\alpha$, IL-2, IL-4 and IL-10), chemokines (RANTES, MCP1, IL-8, MIG and IP-10) and leukotrienes (CysLTs and LTB-4). For each molecule tested, the HTLV-1 individuals were classified as low or high-producers taking the global median index of the HBD group as a cut off.

\section{Results}

When comparing AS and pHAM individuals, AS were high-producers of IP-10 and low-producers of RANTES; pHAM were high-producers of IL-2 and low of IL-8. Besides, AS individuals presented a strong positive correlation between the regulatory cytokines IL-10 with IL-4 and between both with the pro-inflammatory cytokines IL-2 and IL-6; a negative correlation was found between RANTES and IL-2. HAM were high-producers of IL-6, IFN- $\gamma$, IP-10, LTB4, IL-4, MIG, IL-10, IL-2, presented a positive correlation of TNF- $\alpha$ and IFN- $\gamma$ with IL-6, but this group had a positive correlation of CysLT with IL-10, IL-4 and TNF- $\alpha$, contrasting with other groups.

\section{Discussion}

HAM displayed a unique signature of inflammation, which was strengthened by CysLT and not counterbalanced by IL- 4 and IL-10. This signature was observed in pHAM to a lower extent, becoming more evident in HAM. This profile may indicate disease progression and may serve as prognostic markers in future studies.

\begin{abstract}
Authors' details
${ }^{1}$ Curso de Pós-Graduação em Infectologia e Medicina Tropical,Faculdade de Medicina da Universidade Federal de Minas Gerais - Belo Horizonte, Minas Gerais, Brazil. 'Laboratório de Biomarcadores de Diagnóstico e Monitoração, Centro de Pesquisa René Rachou, FIOCRUZ-Minas, Belo Horizonte, Minas Gerais, Brazil. ${ }^{3}$ Fundação Centro de Hematologia e Hemoterapia de Minas Gerais, HEMOMINAS, Minas Gerais, Brazil. ${ }^{4}$ Hospital Sarah Kubitschek, Belo Horizonte, Minas Gerais, Brazil. ${ }^{5}$ Faculdade de Farmácia da USP, Ribeirão Preto, São Paulo, Brazil.
\end{abstract}

Published: 7 January 2014

doi:10.1186/1742-4690-11-S1-037

Cite this article as: Starling et al:: Cytokines, chemokines and

leukotrienes profile and signature analysis in HTLV-1 infection as an evidence of disease progression. Retrovirology 2014 11(Suppl 1):037.

* Correspondence: deniseg@medicina.ufmg.br

'Curso de Pós-Graduação em Infectologia e Medicina Tropical,Faculdade de Medicina da Universidade Federal de Minas Gerais - Belo Horizonte, Minas Gerais, Brazil

Full list of author information is available at the end of the article 